\title{
Fortalecimiento de valores en estudiantes universitarios: su incidencia en la agenda para el desarrollo municipal ${ }^{*}$
}

\section{Values reinforcement for college students: Their incidence in the municipality development agenda}

DOI: https://doi.org/10.17981/juridcuc.16.1.2020.06

Fecha de Recepción: 01/04/2019. Fecha de Aceptación: 01/09/2019

\author{
Blanca Isabel Llamas Félix \\ Universidad Autónoma de Zacatecas (México) \\ blancaisabel@unizacatecas.edu.mx \\ Isabela Scarlett de la Torre Llamas \\ Universidad Autónoma de Zacatecas (México) \\ scarlettritter@gmail.com
Flor de María García Martínez
Universidad Autónoma de Zacatecas (México) florecitagama@hotmail.com \\ Rubén Carlos Álvarez Diez \\ Universidad Autónoma de Zacatecas (México) \\ rubenalvarezdiez@hotmail.com \\ Víctor Hugo Bañuelos García \\ Universidad Autónoma de Zacatecas (México) \\ bag_70@hotmail.com
}

Para citar este artículo:

Llamas, B., de la Torre, I., García, F., Álvarez, R. y Bañuelos, V. (2020). Fortalecimiento de valores en estudiantes universitarios: su incidencia en la agenda para el desarrollo municipal. JURÍDICAS CUC, 16(1). 145-176. DOI: http://dx.doi.org/10.17981/juridcuc.16.1.2020.06

\section{Resumen}

El mundo atraviesa por una gran crisis de valores, lo cual evidencia la necesidad de cambiar el modelo vigente de educación formal, a fin de integrar contenidos programáticos que permitan construir un mundo de valores, planteando la inminente necesidad de reforzar éstos en la educación superior. Como objetivo se analizaron los valores practicados por estudiantes universitarios, así como su incidencia en la agenda para el desarrollo municipal en México, a fin de generar estrategias que les permitan reforzarlos. Se llevó a cabo mediante un enfoque cualitativo, haciendo uso de la entrevista a profundidad y del método descriptivo, empleando la fenomenología, partiendo de una guía de 18 preguntas aplicadas a 100 estudiantes, cuyos resultados fueron analizados pregunta-entrevistados, haciendo hincapié en las respuestas recurrentes, dando pie a que los estudiantes realicen actividades para el desarrollo municipal mediante el involucramiento en labores sociales y realización de proyectos de emprendimiento social.

Palabras clave: Agenda para el desarrollo municipal; estudiantes universitarios; valores
Abstract

The world is going through a great crisis of values, which shows the need to change the current model of formal education, in order to integrate programmatic contents that allow to build a world of values, raising the imminent need to reinforce them in higher education. As an objective, the values practiced by university students were analyzed, as well as their impact on the agenda for municipal development in Mexico, in order to generate strategies that allow them to reinforce them. It was carried out through a qualitative approach, making use of the in-depth interview and the descriptive method, employing phenomenology, based on a guide of 18 questions applied to 100 students, whose results were analyzed question-interview, emphasizing the recurrent answers, allowing students to carry out activities for municipal development through involvement in social work and social entrepreneurship projects.

Keywords: Municipality development agenda; college students; values 


\section{INTRODUCCIÓN}

En los últimos tiempos ha ido surgiendo una nueva crisis, que va desde los lugares más pequeños hasta las grandes potencias mundiales, la cual se ve reflejada en las actividades del día a día y en los diferentes entornos sociales: llámese familiar, de amistad, de estudio, de trabajo o del sector público; donde pareciera acentuarse la problemática al presentarse prácticas corruptas, que ocasionan la falta de credibilidad de los gobernados: la pérdida de valores. Uno de los temas actuales que son promovidos por instituciones públicas y privadas, nacionales e internacionales, es el tema de valores, ya que el hombre es consciente de su deterioro. En tal sentido, los valores de un grupo social, al igual que las instancias que conforman la administración pública, son el instrumento de coordinación y control de la actividad social en aras de obtener un bien colectivo (Ocampo, 2006).

La carencia de valores marcada por la expansión de modernos modos de producción, patrones tecnológicos, la crisis económica global severamente influenciada por la presión del mercado para la maximización de ganancias, la división del trabajo, el intercambio desigual entre naciones, entre muchas otras. Urge a las nuevas generaciones no permanecer impávidos y hacer frente a los nuevos retos que la modernidad y el mundo actual le presentan, reforzando y poniendo de manifiesto sus valores. La educación es un valor inherente a toda cultura, ha existido desde la aparición del hombre en la faz de la tierra, forma parte de su proceso histórico y de su evolución, que por ser parte de la vida, se adquiere durante el desarrollo de ésta y solo termina con la muerte. El ser humano aprende toda su vida, en ocasiones por estímulos ajenos a su voluntad y otras por su propio interés en ello.

El mundo atraviesa por una gran crisis de valores, que pone de manifiesto la necesidad de modificar el modelo educativo actual y contribuir a construir otro que logre el anhelado equilibrio perdido. Siendo aquí donde se detecta la enorme necesidad de modificar la vigente educación formal. La educación se considera un hecho humano 
porque solo el hombre con su razonamiento lo practica y genera su desarrollo con nivel de conciencia y su capacidad de aprendizaje, en un grupo para transformar a la sociedad. El mundo, frecuentemente sin sentido, tiene sed de ideal y de valores, haciendo de la educación la noble tarea de elevar el pensamiento y cierta superación del individuo. La educación durante toda la vida se presenta como una de las llaves de acceso para poder afrontar las novedades que surgen en la vida, se destaca y acentúa la necesidad de volver a la escuela, que sólo puede llevar a buen puerto dicha tarea (Delors, 2008).

La formación del universitario es compleja y delicada, además de los saberes en su campo de conocimiento, han de tener cualidades éticas y morales que le permitan transformar su entorno y contribuir con una sociedad más humana; lo demuestran diferentes investigaciones, tal es el caso realizado con facilitadores del Decanato de Administración y Contaduría de la Universidad Centroccidental Lisandro Alvarado (Venezuela), que al igual que el presente se propuso generar estrategias que coadyuven a la incorporación en las actividades de autodesarrollo de valores, donde los facilitadores coincidieron en considerar la necesidad de incluir estrategias para contribuir en dicha formación (Barón \& Barón, 2013).

En tiempos recientes se ha gestado un importante cambio de modelos educativos para las Instituciones de Educación Superior (IES), implantando modelos que promuevan la formación integral de los educandos, haciendo énfasis en la formación de valores, lo que contribuirá a sus competencias profesionales, mismos que habrán de influir en el futuro profesionista que tendrá que incorporarse de manera efectiva a la vida laboral de la sociedad en la que desee integrarse. Tal modificación tiene como propósito final, el mayúsculo reto de graduar de las instancias universitarias a mejores ciudadanos, tanto en cuestiones de conocimientos técnicos, como a seres humanos que logren contribuir con una sociedad más equitativa y justa que las que se han generado en los últimos tiempos.

El municipio ha sido el mismo desde su origen, aunque en constante evolución, desde las formas más simples de organización hasta las más complejas, pero siempre pendiente de cumplir con su función 
principal: la Administración Pública. Ante el reconocimiento de esta realidad, se hace necesario enfrentar la pérdida de valores desde la educación formal y, como parte de la estructura curricular, a través de asignaturas que permitan incidir en los objetivos propuestos dentro de la agenda de desarrollo municipal, así como en el fortalecimiento de la armonía social. En la Administración Pública los valores son indispensables, los del personal que ahí labora conforman el marco de referencia que guía el pensamiento y la acción de ellos hacia la consecución de los objetivos, cumpliendo una función destacada en el comportamiento de todos. Un administrador público que no actúe bajo parámetros, corrompe y degrada a sus componentes, perdiendo valor y sentido de su razón de ser (Alvarado, 2011).

Investigaciones resaltan el rol de la gestión pública, para el presente la que realiza el municipio, puesto que es el que vincula al Estado con la Ciudadanía, mismo que puede facilitar o inhibir los mecanismos de participación ciudadana, por tratarse de un ente regulador de las relaciones entre los individuos, en un espacio determinado, donde comparten cultura, historia, tradiciones y por supuesto valores, haciendo hincapié en como el ciudadano debe ser participe activo, reconocido con la plenitud de sus capacidades para asumir y enfrentar múltiples variables y situaciones de su entorno e involucrarse en la definición y solución a problemas de su interés (Hernández, Alvarado y Chumaceiro, 2013).

Es por lo antes señalado que el presente trabajo tuvo como objetivo analizar los valores que practican los estudiantes universitarios, así como su incidencia en la agenda de desarrollo municipal en México para generar estrategias que les permitieran reforzarlos. Siendo así que, a lo largo del presente se aborda dicha situación, así como las teorías y conceptos que sustentan la misma, cuya investigación se realizó mediante el enfoque cualitativo, empleando la entrevista a profundidad, usando el método descriptivo, a través de la fenomenología, a partir de una guía de 18 preguntas aplicadas a 100 estudiantes, cuya muestra fue seleccionada al azar, analizando pregunta-respuesta, enfatizando en aquellas que resultaron ser repetitivas. 
Para cerrar el trabajo con las conclusiones y el planteamiento de la urgente necesidad de dar pie a que, tanto docentes como estudiantes, afiancen la educación en valores adquirida durante su infancia, más aún, diseñen estrategias con miras a cumplir dicho propósito, entre las que se encuentren la realización de proyectos de emprendimiento social y su inclusión en las presidencias municipales, con miras a dar cumplimiento a los propósitos de la agenda de desarrollo municipal.

\section{Desarrollo}

En virtud de que la sociedad moderna se encuentra inmersa en una cultura basada en la individualidad, se torna cada vez más difícil observar y practicar principios y valores colectivos, como la solidaridad, la cooperación, el respeto, la responsabilidad, la honestidad y la gratitud. Realidad ante la cual se hace necesario enfrentarla desde la educación formal y como parte de una estructura curricular, con asignaturas que permitan incidir en el fortalecimiento de la armonía social en México. La sociedad civil, las organizaciones públicas y privadas, pero por sobre todo las instituciones educativas, han de unir esfuerzos para construir un mundo de valores, donde ésta última juega un papel trascendente para reorientar pautas de acción y contribuir a la transformación de la sociedad y su entorno; contribuir a la construcción de conocimientos, habilidades, perspectivas y valores, es decir, las competencias necesarias para que los futuros profesionistas participen en sociedad responsablemente.

La sociedad requiere contar con individuos que interioricen los valores y posean una conducta íntegra, ya que las IES, mediante la formación de profesionistas que han de insertarse en el mercado laboral, han de contribuir a la planeación, generación de propuestas y ejercicio cotidiano de valores; a fin de que a través de la inclusión de éstos en las políticas públicas, se logre fortalecer la formación integral de los educandos, que a su vez incidan en la ciudadanía, así como contribuir con la armonía social en México. 
Afirmar que la Administración Pública es más efectiva no deja de ser un simple comentario, al quedar mucho por hacer en esta sociedad cambiante; aún hay que afrontar cambios normativos y de funcionamiento para darle continuidad a su función primordial: el bienestar de la sociedad. La Administración Pública debe darse en un marco de humanización de la realidad, permitiendo que al tiempo que se haga una política pública, no haya barreras que limiten su acción para garantizar el disfrute de su aplicación. Las nuevas políticas públicas tendrán que preocuparse más por afrontar problemas reales de la ciudadanía, esto es, enfrentarse a la mejora con sensibilidad social; ratificando que son los ciudadanos los dueños de la Administración Pública.

Las instituciones educativas y los tomadores de decisiones, generalmente no comparten una misma escala de valores o de intereses, es normal encontrar diferencias o desacuerdos respecto de las políticas públicas a construir y la inminente necesidad de que éstas existan. Para la educación superior sería básico aprender a aprender, hacerlo de manera autónoma, estimular el interés por saber más, con un pensamiento crítico y reflexivo, puesto que en el ámbito laboral se buscan trabajadores capaces de resolver la problemática a que se enfrentan en el día a día las organizaciones, tanto públicas como privadas (Huerta, Pérez y Castellanos, 2000) y en diferentes disciplinas sociales (Luna, 2018; Araque y Suárez, 2017)

El sistema educativo ha de transferir la responsabilidad del aprendizaje a los propios estudiantes, a fin de que sean capaces de establecer metas acordes al contexto social en que se encuentran inmersos y en caso de desviaciones sean capaces de establecer mecanismos correctivos (Méndez, 2005).

Las IES conocen que la motivación dentro y fuera del aula es un factor determinante del aprendizaje, para que los estudiantes resuelvan los problemas que puedan encontrar en su ejercicio profesional. Una característica de la labor en la universidad es la relativa frecuencia con que los alumnos han de hacer trabajos de campo, prácticas, investigaciones, etc. (García-Valcárcel, 2008). 
Una actitud escolar vagamente inspirada en Jean Piaget sostiene que la ética no puede enseñarse de modo temático, como una asignatura más, sino que debe de ejemplarizarse en toda la organización del centro educativo, en las actitudes de los maestros y su relación con los alumnos, así como impregnar el enfoque docente de cada una de las materias (Savater, 1997).

Pareciera que el tema de los valores ha sido relegado a un segundo plano y no ameritar atención especial de parte de las organizaciones encargadas de la planeación de la educación, pero sobre todo, por las instancias gubernamentales. En los últimos años la Administración Pública en México, otros países de América Latina y el resto del mundo, han de enfrentarse a un gran reto: la carencia de valores.

Las IES pueden dar la oportunidad a los estudiantes de integrarse a la realización de trabajos en las presidencias municipales, para contribuir a los objetivos planteados en la agenda para el desarrollo municipal, así como para el fortalecimiento de valores, ya que los trabajos prácticos favorecen el aprendizaje, por lo que se espera que dicha propuesta sea percibida por los estudiantes como positiva (Tapia, 2001).

Partiendo del recorrido anterior se está en condiciones de hacerse los siguientes cuestionamientos: ¿En México, es considerada como obligatoria la educación en valores en el nivel superior? ¿La formación en valores debe ser un ingrediente imprescindible en las políticas públicas diseñadas para las IES? ¿La Administración Pública se ajusta adecuadamente a la demanda ciudadana a través del establecimiento de políticas públicas que contemplen la integración de valores, en busca de la armonía social? ¿La integración de los estudiantes de licenciatura a las presidencias municipales contribuye con los objetivos planteados por la agenda para el desarrollo municipal y la práctica de valores? 
Fundamento TEÓRICO Y CONCEPTUAL

\section{Teórico}

El panorama de estudio sobre el desarrollo de valores ha sido bastante fértil y por tanto existen varias teorías relacionadas con ellos, ya que un asunto tan complejo difícilmente puede ser abordado a la luz de una sola teorización, así que para efectos del presente se toman como soporte teórico las siguientes.

El funcionalismo tomado de la teoría sociológica del estado que señala como la función preponderante en todo sistema social de índole cultural estriba en la adhesión del grupo social a los valores y normas establecidas durante la socialización, además del control social donde las instituciones políticas tienen como función, atender las demandas sociales mediante normas obligatorias para los miembros de la sociedad.

También se encuentra que uno de los propósitos investigativos de Jean Piaget fue el del juicio moral, que según él es un sistema de reglas y la búsqueda del respeto que el individuo adquiere hacia las mismas; lo que para Kant estaba vinculado a la idea del respeto a las personas, para contextualizar el desarrollo moral y el conflicto en el sistema de valores que posee un individuo. Piaget atribuye gran importancia a la educación y declara que sólo ésta puede salvar a la sociedad de una posible disolución, violenta o gradual, por lo que vale la pena luchar. De igual manera se señala la teoría del desarrollo moral de Kohlberg, tan difundida en lo educativo, hasta el punto de convertirse en paradigma moral y punto de referencia obligada en cualquier discusión sobre desarrollo moral y educación en valores.

La crisis del sistema de valores cala de forma profunda en todos los agentes y fuerzas sociales, pero donde se plantea de forma más dramática es en la escuela por efecto de la contradicción y del conflicto de valores que se vive en su seno. La ampliación de los derechos y libertades individuales promovidos y acrecentados por el estado trae consigo la pérdida del sentido de obediencia a toda norma, favoreciendo la indiferencia moral, política y educacional, permitiéndose cosas que deberían ser evitadas (Lauwerys, 1978). 
Junto a los valores, la escuela debe incluir en sus enseñanzas los distintos valores que existen no sólo en la sociedad, sino en el mundo y que forman parte del patrimonio común de la humanidad, y exponer y someter a debate con los alumnos las consecuencias sociales e individuales que tiene la elección de valores (Quintana, 1992).

\section{Conceptual}

\section{- Educación}

A fin de dimensionar el significado de educación, se citan diversos autores en tiempo y espacio.

"La educación consiste en dirigir los sentimientos de placer y dolor hacia el orden ético".

Aristóteles.

"Proceso de adaptación progresiva de los individuos y de los grupos sociales al ambiente, por el aprendizaje valorizado, y que determina individualmente la formación de la personalidad, y socialmente la conservación y la renovación de la cultura".

Bittencourt.

"Educación es evolución, racionalmente conducida, de las facultades específicas del hombre para su perfección y para la formación del carácter, preparándole para la vida individual y social, a fin de conseguir la mayor felicidad posible".

Rufino Blanco.

"La educación es la organización de hábitos de acción capaces de adaptar el individuo a su medio ambiente y social".

James, W.

"La educación consiste en distribuir la cultura, para que el hombre organice sus valores en su conciencia y a su manera, de acuerdo a su individualidad".

Kerschensteiner. 
"La educación es el desarrollo natural, progresivo y sistemático de todas las facultades".

Pestalozzi.

"La función de educar es el proceso de preparar al hombre para la vida completa".

Spencer.

"Educar es transferir a otro, con abnegado amor, la resolución de desarrollar de dentro a fuera, toda su capacidad de recibir y forjar valores".

Spranger.

Educar es contribuir a la formación del hombre en su integridad y este proceso no puede llevarse a cabo sin conciencia. Conciencia de sí, de las propias experiencias y valores, de los actores que los manifiestan, de la existencia de otros seres, que al igual que uno mismo son dignos, libres y responsables (González, 2004).

La educación tiene como objetivo la formación de capacidades y actitudes de los individuos para su integración a la sociedad como seres capaces de regular el statu quo y a la vez puedan transformar la realidad social en pos de los valores vigentes en un momento histórico determinado. Es un proceso a través del cual un individuo modifica su comportamiento respecto a su ambiente de manera planeada, por la acción mediadora, directa o indirecta, que ejerce sobre otro individuo (García, 2004).

Se dice que la educación debe servir para conformar una ética social acorde con los requerimientos del modelo de desarrollo, pero también debe tener fines prácticos: proveer conocimientos, habilidades, capacidades y hábitos para el mundo del trabajo, misma que puede ocurrir en un contexto escolarizado o no (Muñoz, 1996).

De lo anterior se desprende que, la educación es un proceso en constante movimiento, que esencialmente pretende la perfección del hombre, el uso idóneo de sus capacidades, el paso de lo que es a lo que debe ser, el propósito de mejorar al individuo, de su transición 
hacia el ser humano, cambio que conduce a la mejora y transformación continua.

\section{- Valores}

Desde la antigüedad, la palabra "valor", se ha usado para indicar la utilidad de los bienes y el mérito de las personas. Los valores son aquellos principios, virtudes o cualidades que caracterizan a un individuo, acción u objeto, que se consideran típicamente positivos. Son cualidades que se destacan en cada persona y que, le impulsan a actuar, ya que definen la forma de vivir e interrelacionarse con los demás.

Los valores se consideraban implícitos en la tarea educativa. Se daba por sentado que, los maestros al transmitir los contenidos de las diferentes materias, formaban valores; sin embargo, la profunda crisis que afecta a la sociedad occidental ha venido a poner en evidencia la necesidad de proponer internacionalmente la educación en valores, si es que se desea obtener resultados específicos en este campo (Marina y González, 1988).

Los valores siempre apelan a la persona, es decir, están fundamentados en ella, y toda persona, independientemente de su condición, sexo, raza, cultura, educación, creencias... está dotada de un valor por excelencia: su dignidad de carácter intrínseco. Valor sobre el cual reside en la dimensión más genuina y exclusiva de la persona: su dimensión espiritual que otorga unidad, dirección y sentido a todas las demás dimensiones como sus facultades y funciones (Manzano, 2014).

Los valores en la sociedad se transmiten de generación a generación, adquiriendo con ello costumbres, tradiciones, motivaciones, normas de conducta, entre otras; donde cada generación posee su propia escala de valores, en función de prioridades, fines y objetivos que la sociedad marca, que se modifican de manera diferente según la clase, edad, sexo, escolaridad, lugar de residencia, así como la pertenencia a grupos étnicos, religiosos o políticos (Muñoz, 1996). 
Dicen Marina y González (1988), que los valores están en los individuos, que dependen de los sentimientos y experiencias de las personas con respecto de dicho valor, esto es, su valoración; que no se transmiten en forma directa docente-alumno, sino que se aprehenden mediante un proceso propio y único en cada estudiante.

Lo anterior, lleva a que el docente adopte la idea de que la transversalidad de los valores, funciona como mecanismo automático para que los valores en un programa de estudio sean aceptados y aprehendidos por los estudiantes, sin tener en cuenta los aspectos particulares que atrapen a cada estudiante, ni admitir la responsabilidad de descubrir los procedimientos, las técnicas y los métodos adecuadas para las didácticas de los valores (Contreras y Lagunes, 2011).

Los valores tienen gran importancia en el servicio de la administración y las universidades públicas, por el papel que juegan en la transformación de sus actores universitarios (estudiantes, profesores e investigadores) y de las comunidades que interactúan directa e indirectamente en éstas (Alvarado, 2011).

Los valores responden a la armonía entre el actuar y el pensar, esto es, cumplir con lo establecido en la normativa del funcionamiento interno de las universidades, responder al compromiso institucional en correspondencia a las convicciones personales e individuales de los funcionarios públicos, delineándose en función del mismo objetivo, las mismas metas, logrando satisfacción personal y mejora en la calidad del servicio público (Alvarado, 2011).

\section{Educación en Valores}

La educación en valores suscita un gran interés social y educativo hasta el punto de considerar su presencia como un contenido específico en los currículos escolares de todos los niveles educativos. En la última década la educación en valores se ha convertido en el problema estratégico número uno de la educación, se requiere de un amplio debate social para definir los valores que han de regir la conducta colectiva, un empeño de todos los agentes sociales y educativos para hacerlos efectivos, así como de la voluntad para reforzarlos (Parra, 2003) 
Para González (2004) la educación debe ser comprendida como el formar al hombre en su integridad, por lo que debe tener como meta central del proceso, una transmisión sana de valores que faciliten el germinar de lo mejor de las potencialidades humanas.

Mediante la educación, la sociedad tiende a perpetuarse, siendo los valores el medio que da cohesión al grupo, proporcionándoles estándares de vida. La educación es por tanto, aquella actividad cultural que se lleva a cabo en un contexto organizado para transmitir conocimientos, habilidades y valores demandados por un grupo. La educación en valores para la juventud: solidaridad, equidad, convivencia, resolución de conflictos sin violencia, educación emocional y afectiva, desarrollo de actitudes y de las competencias necesarias, así como la orientación para la vida adulta, son ejes fundamentales para el desarrollo integral de las personas, de las relaciones entre ellas y, a su vez, son ejes transversales imprescindibles para la construcción del andamiaje y los pilares de la escuela, junto con la coeducación, la igualdad de oportunidades, de trato, y la prevención de la violencia de género.

La educación y lo que de ella se piensa constituyen un espacio en el que confluyen ideologías, valores y símbolos que son cruciales para estructurar y reestructurar lo económico, ordenar la diferenciación social y darle viabilidad al sistema político, particularmente después de un período de crisis. En la educación se contienen expectativas que dan impulso a la acción para el cambio (Muñoz, 1996).

La educación en valores pretende iniciar con la formulación de las metas establecidas para la educación obligatoria, para que se vayan adquiriendo los valores adecuados, los interiorice y traduzca en proyecto personal de vida que guíe sus obras como individuo y ciudadano. En todo tiempo y lugar, la educación ha contribuido al proceso de socialización de los valores comunes, compartidos por el grupo social, a fin de garantizar el orden social y su continuidad. Los valores, asociados durante mucho tiempo por motivos de ideología con los enfoques humanistas de la educación, en los últimos años se han ido imponiendo como un asunto inherente a los planteamientos de mejora, como exigencia de reconstrucción social. 
El binomio educación y valores, propicia cuestionarse como si se tratase de dos asuntos diferentes, mientras que uno implica a la otra, tanto social como personal y pedagógicamente, pues se sabe que la cuestión antropológica y social del propósito de la acción educativa es la que generar la unidad entre ambos.

\section{Nivel superior}

Hablar de la educación universitaria es un tema complejo, porque desde su aparición, ha experimentado profundos cambios y, sobre todo, ha crecido y se ha multiplicado, es el nivel dedicado a la formación y transmisión de conocimientos al individuo, para el ejercicio de una profesión, es aquella que contempla la última fase del proceso de aprendizaje académico, es decir, aquella que se imparte posterior al bachillerado o equivalente (Parrent, Farrand, Esquivel y Soriano, 2004).

La educación tiene como objetivo la formación de capacidades y actitudes de los individuos para su integración a la sociedad como seres que puedan transformar la realidad social en pos de los valores vigentes en un momento histórico determinado. La tarea de la educación superior es "la formación de profesionales competentes: individuos que resuelvan creativamente, es decir, de manera novedosa, eficiente y eficaz, problemas sociales" (Ibáñez, 1994).

Esta definición reconoce la influencia del contexto del cual se parte, que constituye la meta de transformación y creatividad para la solución de problemas de índole social de manera eficaz y eficiente, transformando las capacidades necesarias en el individuo para el logro de dicha meta, cuya finalidad es proporcionar la capacitación académica para acceder al mercado de trabajo.

La educación superior debe regirse por el rigor intelectual, la libertad de cátedra del profesorado y por valores morales que impregnen el conocimiento académico, incorporando métodos educativos basados en la innovación, así como planteamientos que potencien el pensamiento crítico y la creatividad, dirigida al desarrollo de la creatividad e innovación en si misma, propiciando un ambiente educativo que además de resolver problemas sociales junto a los 
alumnos, también ayude a preparar mejores profesionistas para el futuro.

Las IES son las encargadas de la educación en los jóvenes, las características de éstas están íntimamente relacionadas a la calidad de sus estudiantes, considerando que la calidad hace referencia a un sistema donde los principales factores son los individuos, quienes son capaces de organizarse en forma eficiente para alcanzar las expectativas de la organización educativa (Guerrero, 2003).

La Ley para la Coordinación de la Educación Superior señala (art. 3, 1993):

El tipo educativo superior es el que se imparte después del bachillerato o de su equivalente. Comprende la educación normal, la tecnológica y la universitaria e incluye carreras profesionales cortas y estudios encaminados a obtener los grados de licenciatura, maestría y doctorado, así como cursos de actualización y especialización (Congreso de la República, 1993).

La Educación Superior tiene como finalidad proporcionar formación científica, profesional, humanista y técnica en el más alto nivel, contribuir a la preservación de la cultura nacional, promover la generación y desarrollo del conocimiento en todas sus formas, y desarrollar las actitudes y valores que requiere la formación de personas responsables, con conciencia ética y solidaria, reflexivas, críticas, capaces de mejorar la calidad de vida, consolidar el respeto al medio ambiente, a las instituciones de la República y a la vigencia del orden democrático. La educación superior enfrenta el desafío de fortalecer sus objetivos fundamentales para encontrar equilibrio entre la tarea que se refiere a la búsqueda del conocimiento por sí mismo y la atención a necesidades sociales; fomentar capacidades genéricas o el desarrollo de conocimientos específicos; responder a demandas del empleador o el autoempleo.

La Organización de las Naciones Unidas para la Educación, la Ciencia y la Cultura-UNESCO es la única institución especializada que dispone de un mandato en educación superior y, por tal, facilita la elaboración de políticas de base empírica en materia de 
enseñanza superior, para lo cual ha creado alianzas con dichas instituciones con el objetivo de alentar la cooperación y la creación de redes entre universidades con miras a reforzar las capacidades institucionales mediante el intercambio de conocimientos y el trabajo conjunto (Lemarchand, 2010).

Un nuevo enfoque formativo de las instituciones educativas, de su eficiencia social y pedagógica se ha venido generalizando para dejar claro que la educación es, un proceso de formación moral, es decir, una cuestión de valores. Identificar cuáles son las actitudes de los estudiantes de licenciatura ante el riesgo de sus errores en el abordaje de problemáticas de personas y grupos sociales, que llevan al conocimiento de su ethos académico, concibiendo al ethos como el principal elemento de la disposición de la formación socio moral.

Los centros de educación superior, en el desempeño de sus funciones primordiales deberán concentrarse aún más en los aspectos interdisciplinarios y promover el pensamiento crítico y la ciudadanía activa, contribuyendo así al desarrollo sostenible, la paz y el bienestar, debiendo no sólo proporcionar competencias sólidas para el mundo de hoy y mañana, sino contribuir además a la formación de ciudadanos dotados de principios éticos, comprometidos con la construcción de la paz, la defensa de los derechos humanos y los valores de la democracia (Lemarchand, 2010).

La función de las universidades ha de estar avalada por una formación capaz de dotar a los estudiantes y a los docentes que la generen, de aptitud crítica, cosa que sólo se logrará cuando a la preparación específica se integre la competencia de asumirla; con la formación del pensamiento, la adquisición de valores por parte de los actores de la actividad educativa, es decir, que permita la preparación integral que lleve a los futuros profesionistas a contar con la visión ética necesaria.

\section{Agenda para el Desarrollo Municipal}

El municipio es el lugar donde la relación entre ciudadanía y gobierno, es cotidiana, estrecha y permanente, donde las necesidades 
sociales se hacen presentes, donde los funcionarios municipales viven la responsabilidad de atender de manera eficiente el quehacer de los gobiernos locales, promover un desarrollo con equidad, mediante el impulso de la economía local, el comercio, los servicios públicos y de actividades culturales y recreativas. La sociedad actual necesita gobiernos municipales fuertes y efectivos en su administración para hacer frente a las demandas y contingencias sociales de los tiempos actuales, que realicen mejores prácticas y cuenten con capacidad de conciliación para la solución de problemas municipales (Vega, 2012).

Lo social en estos días se presenta como una constante con un vertiginoso cambio de valores y de las instancias que cotidianamente los transmitían, ahora descalificadas, transformando los problemas sociales en asuntos de naturaleza colectiva. En tal sentido, resulta relevante señalar que el trabajo del municipio se debe realizar en un contexto específico partiendo de éste para ver si coincide o no con los valores, las creencias, las expectativas y las necesidades que determinan la manera de visualizar, interpretar y favorecer una determinada actuación. Así, estar en condiciones de abrir la discusión sobre los elementos que deben contener las políticas públicas en educación superior para contribuir al desarrollo integral con la sociedad en general, así como el impacto social que han de producir éstas.

La agenda surge como un instrumento que tiene como objetivo evaluar e integrar información que permita apoyar y servir de consulta para el buen desempeño del municipio, permitiendo así una mejor organización en sus funciones y, las de las distintas áreas de la administración, con base en su responsabilidad y acorde a los distintos ejes objeto de observancia, entre los que se encuentran el desarrollo territorial, los servicios públicos, la seguridad pública, el desarrollo nstitucional, el económico, el social y el ambiental (Instituto Nacional para el Federalismo y el Desarrollo MunicipalINAFED, 2017).

Cabe señalar que la evaluación surgió en el año 2004, presentada por el INAFED, la cual estuvo vigente hasta el año 2013, teniendo 
por nombre Agenda desde lo Local; de 2014 a 2018, como Agenda para el Desarrollo Municipal; del 2019 a 2024, como Guía Consultiva de Desempeño Municipal, es la misma que impulsa la vinculación con las dependencias y entidades de la Administración Pública Federal. Complemento de la Agenda para el Desarrollo Municipal está la Agenda Ampliada para el Desarrollo Social Municipal, que aunque se ocupa de temas que, en sentido estricto, no constituyen funciones de los municipios, si contribuyen con la federación y los estados en el logro de objetivos de desarrollo integral para sus habitantes.

La Agenda Ampliada para el Desarrollo Social Municipal está integrada por tres ejes temáticos, cada uno de los cuales se subdividen en rubros o temas, que para el presente trabajo se considera el segundo eje, de desarrollo social, mismo que engloba diversos temas, entre los que destaca la educación y por tal, en el presente se abordan aspectos que tienen estrecha relación con su propósito, mismo que no es contribuir a elevar la calidad y cobertura de la educación básica en el municipio, en coordinación con otros órdenes de gobierno, en los últimos tiempos la preocupación de las autoridades por la educación se ha centrado en el nivel superior, con mayor razón a raíz de los severos problemas de desempleo y falta de instituciones que den cabida a los jóvenes.

Una responsabilidad social de la educación superior ante la complejidad de los desafíos mundiales, presentes y futuros, es hacer avanzar la comprensión de problemas polifacéticos con dimensiones sociales, económicas, científicas y culturales, así como la capacidad de hacerles frente. La educación superior debiera asumir el liderazgo social en materia de creación de conocimientos de amplio alcance para abordar retos mundiales, la necesidad de lograr más información, apertura y transparencia en lo tocante a las diversas misiones y actuaciones de cada establecimiento de enseñanza, que pueda cumplir con su cometido gracias a la calidad, pertinencia, eficacia, transparencia y responsabilidad social (Lemarchand, 2010).

Aunado a lo anterior, se suma la agenda 2030 y los objetivos de desarrollo sostenible, que consideran como uno de sus ejes garantizar 
una educación inclusiva, equitativa y de calidad, así como promover oportunidades de aprendizaje durante la vida para todos. Es una agenda civilizatoria, que pone la dignidad y la igualdad de las personas en el centro que requiere de la participación de todos los sectores de la sociedad y del estado para su implementación, que constituirá un apoyo para cada país, mediante el conocimiento de los 17 objetivos de Desarrollo Sostenible asociados a la Agenda (Organización de las Naciones Unidas-ONU, 2018)

\section{Pertinencia del tema}

Ha quedado demostrado que la formación en valores en la educación superior es un tema necesario, porque su estado predominante en la sociedad actual demanda con urgencia profesionales que, sean capaces de generar un nuevo clima de valores en su entorno cercano. Si bien es cierto que en la formación en valores de la persona involucra a varios actores -familia, escuela, ambiente cultural, político y social, entre otros- no cabe duda que un lugar importante para esta formación de la persona es la educación superior (Manzano, 2014).

\section{MetodoloGía}

La presente investigación se planteó desde un enfoque cualitativo, en virtud de que concibió a la educación como un proceso social históricamente determinado, capaz de ser observado desde una perspectiva específica que generó datos, ya que dicho enfoque se basa en métodos de recolección de datos sin medición numérica, como las descripciones, es flexible y se mueve entre los eventos y su interpretación. Así mismo, por tratarse de un enfoque que proporciona profundidad a los datos, dispersión, riqueza interpretativa, contextualización del ambiente o entorno, detalles y experiencias únicas; aportando un punto de vista fresco, natural y holístico de los fenómenos (Hernández, Fernández y Baptista, 2010). El enfoque cualitativo se sustenta en una postura filosófica postpositivista, al no poder ser positivo acerca de las concepciones de conocimientos 
cuando se estudia la conducta y las acciones humanas (Creswell, 2007), como es el caso del presente trabajo.

Tiene un carácter descriptivo, ya que selecciona una serie de cuestiones y colecta información sobre cada una de ellas para describir lo que investiga, ofreciendo la posibilidad de realizar predicciones o establecer relaciones, aunque sean poco elaboradas (Danhke, 1989); en este caso las cuestiones principales a que alude son: los valores, el contexto, procesos de implementación de los mismos y su contribución a los propósitos de la agenda ampliada de desarrollo social municipal.

Se utilizó la fenomenología, en virtud de que el investigador identifica la esencia de las experiencias humanas respecto del fenómeno, comprende experiencias vividas, es descrita por los participantes en el estudio, analiza un pequeño número de sujetos y el investigador limita sus propias experiencias para comprender las de los participantes (Tashakkori \& Creswell, 2007). De igual manera tuvo un carácter descriptivo y de campo, ya que tratan sobre estudios que buscan especificar propiedades, características y perfiles importantes de personas, grupos, comunidades o cualquier otro fenómeno que se someta a un análisis, que únicamente pretenden medir o recoger información de manera independiente o conjunta sobre los conceptos o variables a que se refieren.

Respecto de los participantes y su contexto se llevó a cabo dentro de diferentes aulas de las instalaciones de la Unidad Académica de Contaduría y Administración de la ahora Benemérita Universidad Autónoma de Zacatecas. La selección de los alumnos muestra se realizó al azar, colocando todas las matrículas de la población, en forma aleatoria para posteriormente seleccionar los primeros 100 integrantes.

El número de muestra se determinó con la siguiente fórmula (Hernández, Fernández y Baptista, 2010):

$$
n=\frac{p(1-p)}{h 2 p(1-p) Z 2 N}
$$


Donde:

$n=$ Tamaño de la muestra

$p=$ Probabilidad de ocurrencia del evento

(Por lo regular se utiliza un valor de 0.3 a 0.5 )

$h=$ Precisión deseada para el estudio (Por lo regular $+/-5 \%$

o sea 0.05 y para este caso se utilizó 0.060)

$Z=\quad$ Coeficiente de confiabilidad

(Por lo regular se utiliza el 95\%,

lo que equivale a un valor de 1.96)

$N=$ Tamaño de la población

Tamaño de la muestra 100 alumnos.

El instrumento de recolección de datos utilizado fue la entrevista a profundidad, para lo cual se elaboró una guía de 18 preguntas, llevada a cabo en diferentes horarios de clase de los involucrados, complementada con la observación durante la realización de la misma. Durante la entrevista se estableció un protocolo de observación para el registro de la información, se tomó nota de las expresiones faciales, los movimientos corporales, las pausas y modulaciones de voz, en el entendido de que se trata de formas utilizadas para la expresión no verbal de sentimientos, emociones, percepciones, aprobaciones y desaprobaciones. Se llevó a cabo una vez logrado crear un clima favorable para la misma, previamente habiendo comentado la forma de llevarla vía palabras iniciales, se plantearon las preguntas clave y se realizó el registro de notas reflexivas sobre la misma.

Para analizar los datos recolectados, se tomó la decisión de estandarizar las categorías propuestas, con las diferentes respuestas proporcionadas por los entrevistados, haciéndolo de manera indistinta y sin seguir el orden establecido en ellas, puesto que el factor que determinó el orden fue justamente las palabras relacionadas con el aspecto de estudio específico. 


\section{Resultados-Discusión}

En virtud de que el análisis en el enfoque cualitativo no es lineal, que se sabe dónde comienza, pero no dónde habrá de terminar, se formularon una serie de preguntas a través de entrevistas abiertas, para lo cual se estructuró el instrumento a partir de la Tabla 1. Dicho instrumento se aplicó a 100 estudiantes de Licenciatura de la Unidad Académica de Contaduría - UAC y Administración de la Universidad Autónoma de Zacatecas - A-UAZ (Bañuelos, Dueñas, Jara, Ramírez y Vital, 2018), iniciando por el significado que cada alumno le asigna al término "valores", encontrándose una gran diversidad de respuestas, como lo son las siguientes: se determinó que los estudiantes fueron repetitivos en su forma de conceptualizarlos, no obstante consideraron la importancia de la buena voluntad, buenas costumbres, observancia de las reglas y principios.

TABLA 1.

Entrevista aplicada a estudiantes de nuevo ingreso UAC y A-UAZ 2018.

\begin{tabular}{|c|c|c|}
\hline No. & Pregunta & Propósito de la pregunta \\
\hline 1. & ¿Para usted qué son los valores? & Conocimiento de los valores. \\
\hline 2. & $\begin{array}{l}\text { ¿Considera que en la actualidad } \\
\text { se aplican los valores? De ser así, } \\
\text { ¿dónde cree usted que se aplican? }\end{array}$ & $\begin{array}{l}\text { Conocer si los valores se aplican y } \\
\text { dónde. }\end{array}$ \\
\hline 3. & $\begin{array}{l}\text { Si considera que en la actualidad no } \\
\text { se aplican los valores, ¿qué medidas } \\
\text { recomienda sean tomadas a fin de } \\
\text { que se observen en la actualidad? }\end{array}$ & $\begin{array}{l}\text { Proponer sugerencias para la } \\
\text { aplicación de los valores. }\end{array}$ \\
\hline 4. & ¿Para usted qué es el respeto? & Conocimiento del valor respeto. \\
\hline 5. & $\begin{array}{l}\text { ¿Considera que en la actualidad } \\
\text { existe el respeto? De ser así, ¿dónde } \\
\text { cree que exista? }\end{array}$ & $\begin{array}{l}\text { Conocer si el valor respeto se aplica } \\
\text { y dónde. }\end{array}$ \\
\hline 6. & $\begin{array}{l}\text { Si considera que en la actualidad } \\
\text { no existe respeto, ¿qué medidas } \\
\text { recomienda sean tomadas a fin de } \\
\text { que exista en la actualidad? }\end{array}$ & $\begin{array}{l}\text { Proponer sugerencias para la } \\
\text { aplicación del valor respeto. }\end{array}$ \\
\hline 7. & $\begin{array}{l}\text { ¿Para usted qué es la } \\
\text { responsabilidad? }\end{array}$ & $\begin{array}{l}\text { Conocimiento del valor } \\
\text { responsabilidad. }\end{array}$ \\
\hline
\end{tabular}


¿Considera que en la actualidad

8. existe responsabilidad? De ser así,

¿dónde cree usted que existe?

Si considera que en la actualidad

9. no existe responsabilidad, ¿qué medidas recomienda sean tomadas a fin de que exista en la actualidad?

10. ¿Para usted qué es la honestidad?

$¿$ Considera que en la actualidad

11. existe honestidad? De ser así,

¿dónde cree usted que existe?

Si considera que en la actualidad

12. no existe honestidad, ¿qué medidas recomienda sean tomadas a fin de que exista en la actualidad?

13. ¿Para usted qué es la solidaridad?

¿Considera que en la actualidad

14. existe solidaridad? De ser así,

¿dónde cree usted que existe?

Si considera que en la actualidad

15. no existe solidaridad, ¿qué medidas recomienda sean tomadas a fin de que exista en la actualidad?

16. ¿Para usted qué es la gratitud?

¿Considera que en la actualidad

17. existe gratitud? De ser así, ¿dónde cree usted que existe?

Si considera que en la actualidad

18. recomienda sean tomadas a fin de que exista en la actualidad?

Conocer si el valor responsabilidad se aplica y dónde.

Proponer sugerencias para la aplicación del valor responsabilidad.

Conocimiento del valor honestidad.

Conocer si el valor honestidad se aplica y dónde.

Proponer sugerencias para la aplicación del valor honestidad.

Conocimiento del valor solidaridad.

Conocer si el valor solidaridad se aplica y dónde

Proponer sugerencias para la aplicación del valor solidaridad.

Conocimiento del valor gratitud.

Conocer si el valor gratitud se aplica y dónde

Proponer sugerencias para la aplicación del valor gratitud.

Fuente: Elaboración propia a partir de Llamas (2017).

Cabe mencionar que los valores se perciben en las actuaciones de los otros, en las relaciones de cada uno con el resto; cada persona, debe construir su propio esquema de valores y la función de los educadores es colaborar en el proceso, permitiendo y desarrollando situaciones en el entorno de los alumnos para que los vivan y experimenten, y así, ser interiorizados por ellos (Parra, 2003). 
Respecto a si los estudiantes conocen la existencia y empleo de los valores y, de ser así, saber dónde se aplican, las respuestas fueron abismalmente opuestas, esto es, desde quienes aseveraron su existencia y aplicación hasta los que hablaron de la inminente desaparición de los mismos. Siendo así: los que aseguraron que los valores si existen, fueron muy enfáticos en indicar que básicamente son dos lugares donde es inminente su sobrevivencia, siendo éstos: la casa y la escuela (Parra, 2003). Para en un aula percibir los valores y su necesidad, han de presentarse ciertos requisitos que posibiliten y alienten su desarrollo.

El tercer cuestionamiento tuvo como propósito que el estudiante, cuya respuesta hubiese sido negativa respecto de la existencia de los valores, propusiera algunas medidas de solución a la respectiva problemática, mediante indicación expresa de dicha respuesta, siendo así que se encontraron las siguientes: los alumnos consideraron que deben enseñarse en el hogar y reforzarse en las instituciones educativas, partiendo del supuesto de que solo le tocará reforzar y no inculcar, ya que para ellos la enseñanza debe ser en la casa. Examinando las respuestas proporcionadas, se aprecia que los estudiantes identificaron algunos rasgos relacionados con la conceptualización, pero que tuvieron problema para proporcionar la definición, facilitándoseles más proveer algunos ejemplos.

Analizando la totalidad de las respuestas y las anteriormente registradas, habrá que insistir en la dificultad representada para los estudiantes en conceptualizar los valores, lo que hace suponer que desconocen el verdadero significado.

La palabra respeto proviene del latín respectus y significa atención o consideración. Es un valor que permite al ser humano reconocer, aceptar, apreciar y valorar las cualidades del prójimo y sus derechos. Es el reconocimiento del valor propio y de los derechos de los individuos y de la sociedad (Mézerville, 2004). Conocer el sentir del estudiante de licenciatura respecto de la existencia y empleo del respeto, y de ser así saber dónde consideran que se aplican: la cantidad de respuestas a favor fue prácticamente nula, en realidad se habla de dos o tres personas que consideraron que sí existe y se aplican en casa, 
escuela y empresa, mientras que el resto simplemente indicó desconocer si se emplea y en su caso dónde. Cabe mencionar que tanto para la conceptualización como para las propuestas para el rescate del valor "respeto", los estudiantes presentaron gran dificultad, lo cual pudo deberse a que los entrevistados fueron de grupos de alumnos que recién comenzaron su formación profesional, y tuvieron problema para expresar sus ideas y proporcionar respuesta en el sentido que se planteó la pregunta.

Responsabilidad significa responder, dar respuesta al llamado de otro. Está íntimamente ligada a la vocación, palabra proveniente del latín vocatio o acción de llamar. Tiene que ver con cumplir con las obligaciones personales, familiares, laborales y ciudadanas; con obedecer a la propia conciencia y asumir las consecuencias de nuestras decisiones y acciones (Trujillo, s.f.). En relación al valor "responsabilidad" y si se aplica: se concluyó que para los estudiantes, la propuesta giró en torno a que sean inculcados en el seno familiar, en las escuelas, mediante conferencias y capacitación.

Estar al tanto de si el alumno sabe el significado de "honestidad" y de ser así, saber dónde considera que se aplica, comentaron que en casa, escuela y calle, claro está, en un mínimo de respuestas afirmativas (González, 2000). Es una cualidad humana que consiste en comportarse y expresarse con coherencia y sinceridad, como el simple respeto a la verdad en relación con el mundo, los hechos y del sujeto consigo mismo. Con las respuestas proporcionadas se pudo apreciar la dificultad al definir y al desconocimiento que sobre el tema tienen, también se percibió confusión con otro concepto estrechamente relacionado, como lo es la honradez (Vargas, 2006). La define como la cualidad de la persona que obra y actúa con rectitud, justicia y honestidad, se deriva de honrado, participio del verbo honrar, se basa en el respeto al otro y en la valoración de la verdad como un valor fundamental de la vida en sociedad.

De acuerdo a (Groser, 2014) la solidaridad es la obligación recíproca de los miembros de grupos u organizaciones para apoyarse unos a otros y ayudarse mutuamente. Conocer el sentir del estudiante de licenciatura respecto de la existencia y empleo de la gratitud y, 
de ser así, saber dónde consideran que se aplica, al igual que hacer propuestas de solución a la problemática que representa su ausencia, una vez manifestado el considerar que no existe. Al igual que en todos los cuestionamientos anteriores, destaca la dificultad que mostraron los entrevistados, evidenciando la gran ignorancia que tienen respecto de todos y cada uno de los valores.

\section{Conclusiones}

La educación se ha dado desde tiempo atrás y, por consiguiente, se ha concebido como un medio para dar identidad, para impulsar los valores, comprender y solucionar los problemas del país. Reforzar la educación y con ella los valores, hace fuertes y permite rechazar prejuicios que impiden conocer la verdad. Enseñar y practicar los valores hacen que la educación responda al interés general de la sociedad porque estimula y promueve nuestro bienestar y el mejoramiento económico, social y cultural de todos.

Partiendo del objetivo planteado en el presente escrito, es decir analizar los valores que practican estudiantes universitarios, se concluyó que éstos no son capaces ni de conceptualizarlos ni de vivirlos en el día a día, es decir, las instituciones educativas han fallado en su labor de inculcar valores en los infantes y, por tanto, llegan al nivel superior carentes de ellos o en el mejor de los casos haciéndoles caso omiso. Si bien es cierto que el aprendizaje corresponde a la etapa inicial de vida, también lo es que aquello que se olvida es necesario reforzarlo recurrentemente, ya que son los propios estudiantes los que reconocieron la importancia de retomar la educación en valores aun en educación superior, argumentando que si éstos no se observaran el mundo difícilmente se encontraría como es que lo está.

Por otro lado, se encontró que ya que parte del objetivo planteado fue conocer de la incidencia del estudiante universitario en la agenda para el desarrollo municipal en México, a través de la generación de estrategias que les permitieran reforzar sus valores, se concluyó que es factible incidir en la misma mediante el establecimiento de convenios de colaboración con el municipio, para que los estudian- 
tes realicen prácticas profesionales en ellos, pero específicamente dentro de los objetivos planteados en la agenda para el desarrollo municipal.

Haciendo notar que desde 2017, los alumnos de la Benemérita Universidad Autónoma de Zacatecas (México) han logrado la vinculación con los municipios, además de venir realizando labores tales como organización de eventos para ayudar a la Asociación Mexicana en Ayuda a Niños con Cáncer (AMANC), con colectas para financiar diálisis en el Hospital General de Zacatecas y organizar conferencias para sensibilizar sobre la donación de sangre, conducir con responsabilidad sin alcohol, fomento a la lectura y prevención del suicidio, entre muchas más. De igual manera los estudiantes han estado generando proyectos de emprendimiento social en vinculación con el municipio, tal es el caso de investigaciones para el establecimiento de pequeños negocios sustentables, con impacto económico, ambiental y/o social. Lo que ha logrado incidir favorablemente en el fortalecimiento de los valores de los estudiantes involucrados en dichas actividades.

De igual manera se considera que la propuesta que plantea la presente investigación, no será una tarea fácil, sin embargo se espera que sea el municipio el que promueva la inclusión de la misma en la formación profesional, a fin de buscar la armonía social y el retorno a la vida armoniosa de antaño, que solo requiere apoyarse en los valores para desterrar las nuevas tendencias de aquellos que reconocen desconocer su presencia en la cotidianidad de la vida.

\section{REFERENCIAS}

Alvarado, L. (2011). Reflexiones teórias: Valores éticos en la prestación de servicios de la administración pública. Gestión y Gerencia, 5(2), 31-58.

Araque, F. y Suárez, O. (2017). Reflexiones teóricas y legales del adulto mayor y la discapacidad en Colombia. Juridicas CUC, 13(1), 97-120. https://doi.org/10.17981/juridcuc.13.1.2017.05 
Bañuelos, S. J., Dueñas, D., Jara, M. M., Ramírez, M. E. y Vital, S. L. (2018). Entrevista aplicada a estudiantes de nuevo ingreso UAC y A-UAZ 2018. [Entrevista]. Universidad Autónoma de Zacatecas, México.

Barón, L. y Barón, N. (2013). Propuesta de una universidad didáctica para la educación en valores según el método antropológico en el marco de una actividad de autodesarrollo. Gestión y Gerencia, 7(1), 48-65.

Contreras, J. y Lagunes, A. (2011). Las didácticas de los Valores en la educación superior, un reto para la formación integral del estudiante. En, XII Congreso Internacional de Teoría de la educación. CITE 2011, Universitat de Barcelona, España.

Danhke, C. P. (1989). Investigación y comunicación. Madrid: McGraw Hill.

Delors, J. (2008). La Educación enciera un tesoro. Informe a la UNESCO de la Comisión Internacional sobre la Educación para el Siglo XXI. [ED.96/WS/9]. UNESCO. Recuperado de https://unesdoc.unesco.org/ark:/48223/pf0000109590_ spa

Estados Unidos Mexicanos. Congreso de la Rep[ublica. (13 de julio de 1993). Ley para la Coordinación de la Educación Superior. [Acuerdo 18/11/18]. DOF: 27/11/2018. Disponible en http://www.ordenjuridico.gob.mx/Documentos/Federal/ html/wo39036.html

García-Valcárcel, A. (2008). La tutoría en la enseñanza universitaria y la contribución de las TIC pra su mejora. RELIEVERevista Electrónica de Investigación y Evaluación Educativa, 14(2), 1-14. Recuperado de http://www.uh.cu/static/ documents/TD/La\%20Tutoria\%20ensennanza\%20universitaria.pdf

González, A. M. (2004). El enfoque centrado en la persona, aplicaciones a la educación. México, D. F.: Trillas.

González, D. (2000). Los valores y su formación: una interpretación psicológica. Revista Cubana de Psicología, 17(3), 307-311. 
Groser, M. (2014). Los principios de solidariad y subsidiariedad. En, Sánchez, H. (ed.), Antologías para el estudio y la enseñanza de la ciencia política. Volumen I: Fundamentos, teoría e ideas políticas, (1657-165). México, D.F.: UNAM. Disponible en https://biblio.juridicas.unam.mx/bjv/detallelibro/3710-fundamentos-teoria-e-ideas-politicas

Guerrero, J. (2003). Calidad en la educción, organizaciones y ejercicio profesional. Alternativas en Psicología, 8, 24, 35.

Hernández, R., Fernández, C. y Baptista, P. (2010). Metodología de la investigación. (5 Ed.). México, D.F.: McGraw-Hill.

Hernández, J., Alvarado, L. y Chumaceiro, A. C. (2013). Estado, gestión pública y participación ciudadana desde la modernidad y postmodernidad. Gestión y Gerencia, 7(1), 66-80.

Huerta, P. Pérez, I. y Castellanos, A. (2000). Desarrollo curricular por competencias profesionales integrales. [Online]. Recuperado de https://www2.ufro.cl/docencia/documentos/Competencias.pdf

Ibáñez, B. C. (1994). Pedagogía y Psicología Interconductual. Revista Mexicana de Análisis de la Conducta, 20(1), 99-112.

INAFED. (2017). Programa Agenda para el Desarrollo Municipal. [Online]. Disponible en https://www.gob.mx/inafed/ documentos/programa-agenda-para-el-desarrollo-municipal-2017-166983

Lauwerys, J. (1978). Some Thoughts on Moral Education. A Think Paper Prepared for the International Council for Educational Development. Connecticut: Essex.

Lemarchand, G. A. (ed.) (2010). Sistemas nacionales de ciencia, tecnología e innovación en América Latina y el Caribe. Uruguay: UNESCO. Disponible en https://unesdoc.unesco.org/ ark:/48223/pf0000187122

Llamas, B. I. (2017). Administración Pública y la necesidad de replantear políticas que integren la educación en valores al nivel superior. [Tesis]. Univesidad de Zacatecas, Zacatecas, México. 
Luna, F. (2018). El mito del cientificismo en la valoración de la prueba científica. Jurídicas CUC, 14(1), 119-144. https:// doi.org/10.17981/juridcuc.14.1.2018.06

Manzano, M. (2014). La formación en valores en la educación superior a distancia. El caso de la Unicersidad técnica particular de Loja. [Tesis doctoral]. UNED, Ecuador.

Marina, A. P. y González, M. Á. (1988). Clarificación de valores y desarrollo humano, estrategias para la escuela. Madrid: Narcea.

Méndez, C. (2005). La implantación del sistema de creditos europeos como una oportunidad para la innovación y mejora de los procedimientos de enseñanza aprendizaje en la Universidad. Revista Española de Pedagogía, 63(230), 5-16.

Mézerville, G. D. (2004). Ejes de salud mental. Los procesos de autoestima, dar y recibir afecto y adaptación al estrés. México, D.F.: Trillas.

Muñoz, H. T. (1996). Los valores educativos y el empleo en México. México, D.F.: Porrúa.

Ocampo, P. M. (2006). Valores universitarios en la actualidad y su perspectiva hacia el futuro. Revista Mexicana de Filosofía "Per Se» [Conferencia pronunciada en la ciudad de Torreón].

ONU. (2018). Agenda 2030 y los Objetivos de Desarrollo Sostenible: una oportunidad para América Latina y el Caribe. [Online]. Santiago: CEPAL. Recuperado de https://repositorio. cepal.org/handle/11362/40155

Parra, J. M. (2003). Educación en valores y su práctica en el aula. Tendencias Pedagógicas, 8(1), 66-87.

Parrent, J. M., Farrand, J., Esquivel, N. H. y Soriano, R. (2004). ¿Qué es la Universidad? México, D.F.: Universidad Autónoma de México.

Quintana, J. M. (1992). Educación en valores y diseño curricular. En, J. García et al., Perfiles para una nueva educación, (15-26). Granada: Universidad de Granada.

Savater, F. (1997). El valor de educar. Barcelona: Ariel. 
Tapia, J. (2001). Motivación y estrategias de aprendizaje. Principios para su mejora en alumnos universitarios. En, A. García (Coord.) Didáctica Universitaria, (79-112). Madrid: La Muralla.

Tashakkori, A. \& Creswell, J. W. (2007). Differing Perspectives on Mixed Methods Research. Journal of Mixed Methods Research, 1(4), 303-308 https://doi. org/10.1177/1558689807306132

Trujillo, J. (s.f.). Responsabilidad "Valor Universitario». [Online]. Disponible en web.uaemex.mx/identidad/docs/RESPONSABILIDAD.pdf

Ulizama, J. (2004). Tecnologías multimedia en el ámbito educativo. [Online]. Recuperado de https://recyt.fecyt.es/index.php/pixel/article/viewFile/61110/37124

Vargas, D. (2006). El Valor y su origen dentro de la Esencia Humana. A Paste Rey 45, 1-8.

Vega, M. M. (2012). Guía práctica del enlace de la agenda desde lo local. San Luis Potosí: Coordinación Estatal para el Fortalecimiento Institucional de los Municipios.

\footnotetext{
* Este trabajo forma parte de la tesis "Administración pública y la necesidad de replantear políticas que integren la educación en valores al nivel superior".
}

Blanca Isabel Llamas Félix es doctora en Educación por la Universidad Autónoma de Durango Campus Zacatecas (México). Doctora en Administración Pública por el Instituto Internacional del Derecho y del Estado (México). Maestra en Administración por la Universidad Autónoma de Zacatecas (México). Licenciada en Contaduría por la Universidad Autónoma de Zacatecas (México). Docente-Investigador de la Unidad Académica de Contaduría y Administración de la Universidad Autónoma de Zacatecas (México). https://orcid.org/0000-0002-0782-8340 
Isabela Scarlett de la Torre Llamas es pasante de la Licenciatura en Letras por la Universidad Autónoma de Zacatecas (México). Pasante de la Licenciatura en Psicología por la Universidad Autónoma de Zacatecas (México). Mentor de la Universidad Autónoma de Zacatecas. https://orcid.org/0000-0001-8881-4161

Flor de María García Martínez es docente-Investigador de la Unidad Académica de Psicología de la Universidad Autónoma de Zacatecas (México). https://orcid.org/0000-0003-3869-0169

Rubén Carlos Álvarez Diez es Doctor en Administración Pública por el Instituto Internacional del Derecho y del Estado (México). Magíster en Mercadotecnia y Negocios Internacionales por la Universidad Autónoma de Durango (México). Docente-Investigador de la Unidad Académica de Contaduría y Administración de la Universidad Autónoma de Zacatecas (México). https://orcid.org/0000-00020877-2238

Víctor Hugo Bañuelos García es Ingeniero por la Universidad Autónoma de Zacatecas (México). Docente-Investigador de la Unidad Académica de Contaduría y Administración de la Universidad Autónoma de Zacatecas (México). https://orcid.org/0000-0003-0888-4157 